\title{
Bayesian Analysis for Step-Stress Accelerated Life Testing using Weibull Proportional Hazard Model
}

\author{
Naijun Sha • Rong Pan
}

Received: date / Accepted: date

\begin{abstract}
In this paper, we present a Bayesian analysis for the Weibull proportional hazard (PH) model used in step-stress accelerated life testings. The key mathematical and graphical difference between the Weibull cumulative exposure (CE) model and the PH model is illustrated. Compared with the CE model, the PH model provides more flexibility in fitting step-stress testing data and has the attractive mathematical properties of being desirable in the Bayesian framework. A Markov chain Monte Carlo algorithm with adaptive rejection sampling technique is used for posterior inference. We demonstrate the performance of this method on both simulated and real datasets.
\end{abstract}

Keywords Step-stress accelerated life test · Proportional hazard model · Cumulative exposure model · Weibull distribution · Bayesian inference

\section{Introduction}

Accelerated life testing (ALT) is often used for making reliability prediction because it collects failure data quickly and reduces the cost of testing greatly. To hasten the process of failure, the testing involves physical stresses at some higher levels than the stress level that the product would experience at its use condition. Step-stress ALT (SSALT) is a special kind of ALT in which the stress on each specimen is increased step-by-step over time. The goal of statistical inference for step-stress ALT is to predict the product failure behavior under the use condition by using the failure data obtained under elevated test conditions.

There are two different ways of modeling SSALT in general. One assumes that the effect of varying stress shortens or expands the product's lifetime depending on the degree of increase or decrease of stress level. This is the tampered random variable (the product lifetime) model (TRV) proposed by DeGroot and Goel (1979). Doksum and Hoyland (1991) brought forth a tampered lifetime model from the decay process point of view. Alternatively, Nelson's cumulative exposure model (CE), which has a significant influence in engineering applications, assumes that the remaining life of test units depends only on the current cumulative fraction failed and current stress, and it has nothing to do with the way of cumulation (Nelson, 1980). It can be shown that the CE model coincides with the TRV model if the product life

\footnotetext{
Naijun Sha

Department of Mathematical Sciences, University of Texas at El Paso, TX 79968

Tel.: (915)747-6844, E-mail: nsha@utep.edu

Business Information Management School, Shanghai Institute of Foreign Trade, Shanghai 201620, China

Rong Pan

School of Computing, Informatics, and Decision Systems Engineering, Arizona State University, AZ 85287

Tel.: (480)965-4259, E-mail: Rong.Pan@asu.edu
} 
distributions under different stresses belong to the same location-scale parametric family (Wang and Fei, 2004). As most of the commonly used distributions in reliability engineering, such as exponential, Weibull and lognormal distributions, belong to log-location-scale families, in practice, there is no difference of modeling SSALT using TRV or CE model.

The other type of SSALT model assumes that the effect of varying stress applies on the hazard function of the product's lifetime. In particular, the Cox's proportional hazard (PH) (Cox, 1972) assumption states that the ratio of hazard functions under two constant stresses is an exponential function of the difference of the two stress levels, i.e. the hazard (or failure rate) function at the stress level $x$ is given by $h(t ; x)=h_{0}(t) e^{x \beta}$, where $h_{0}(t)$ is a baseline hazard function. It is well known that the parameter $\beta$ is the effect of stress, and the baseline hazard function does not require a specific form; therefore, the $\mathrm{PH}$ model provides flexibilities in modeling lifetime data. In fact, many physical failure acceleration models correlate the effect of physical stress to the failure rate of the product, instead of the failure time. For examples, in the Arrhenius temperature acceleration model, the product failure rate is a log-linear function of the proportional inverse of temperature in degree Kelvin; in the Peck humidity acceleration model, the failure rate is a log-linear function of the logarithm of the relative humidity. Although in a constant stress testing, when the failure time has a Weibull distribution, the CE model can be reparameterized as a proportional hazard (PH) model (Lawless, 2003), and this is not true for other distributions or nonconstant stress testings. Therefore, it is necessary to study the statistical inference method of PH-SSALT model for engineering applications where the acceleration model is a failure rate function.

Alternatively, Weibull CE model has been widely studied for SSALT in the past few years. Lee and Pan (2008) described the Bayesian inference of the log-linear failure rate acceleration function for a two-step SSALT when failure time is exponentially distributed (a special case of Weibull distribution). A Bayesian approach to analyzing varying-stress reliability tests using the exponential and Weibull CE models was presented by Dorp and Mazzuchi $(2004,2005)$. The mathematical intractability of likelihood function of CE model makes statistical inference extremely difficulty, even for exponential random failure time (Dorp and Mazzuchi, 2004). Khamis and Higgins (1998) proposed an alternative model to the Weibull CE model, that is, Weibull proportional hazard model (Weibull PH model) although they did not call this name, and presented a maximum likelihood estimation for statistical inference. This PH model have certain analytical advantages over the $\mathrm{CE}$ model in terms of modeling flexibility and computational simplicity. This is particularly desirable for Bayesian analysis.

In this article, we develop a Bayesian inference procedure for the SSALT parameter estimation using Weibull PH model. In Section 2 we review the Weibull PH model and discuss the common ground and differences between this model and the Weibull CE model. We describe the Bayesian approach and the MCMC procedure in Section 3. In Section 4 we assess the performance of the method on simulated datasets and real problems. Finally we conclude the article with a brief discussion in Section 5 .

\section{Weibull Proportional Hazard Model}

Khamis and Higgins (1998) presented a model (KH model) based on a time transformation of the exponential CE model, where the cumulative distribution function (cdf) of failure time $w$ in SSALT is given by

$$
F(w)=1- \begin{cases}\exp \left\{-\theta_{1} w^{\delta}\right\} & 0 \leq w<\tau_{1} \\ \exp \left\{-\theta_{2}\left(w^{\delta}-\tau_{1}^{\delta}\right)-\theta_{1} \tau_{1}^{\delta}\right\} & \tau_{1} \leq w<\tau_{2} \\ \cdots & \\ \exp \left\{-\theta_{k}\left(w^{\delta}-\tau_{k-1}^{\delta}\right)-\ldots-\theta_{2}\left(\tau_{2}^{\delta}-\tau_{1}^{\delta}\right)-\theta_{1} \tau_{1}^{\delta}\right\} & \tau_{k-1} \leq w<\infty\end{cases}
$$

where $\tau_{1}, \ldots, \tau_{k-1}$ are the time points of changing stress levels. Without loss of generality, we assume that stress levels, $x_{i}$ 's, are arranged in an increasing order, i.e., $x_{1}<x_{2}<\ldots<x_{k}$. Note that when $\delta=1$ the above function becomes the cdf of SSALT with exponential distribution. The parameter $\theta_{i}$ is related to 


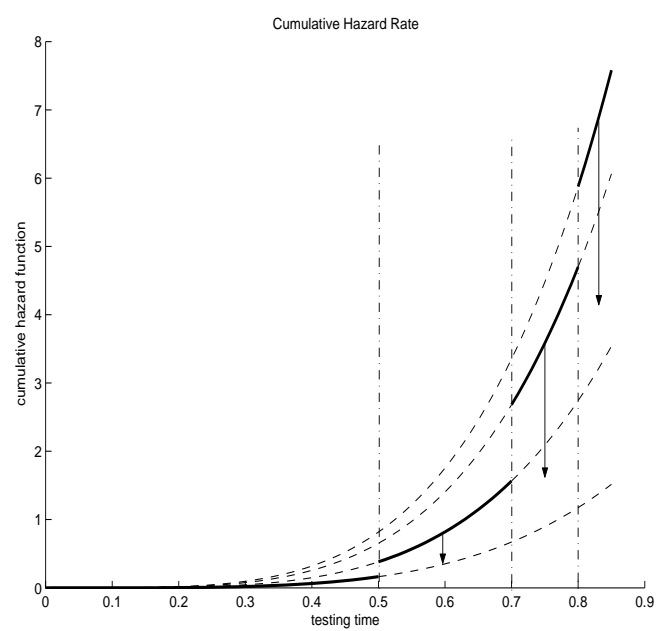

(a) Cumulative Hazard Functions of ConstantStress ALTs

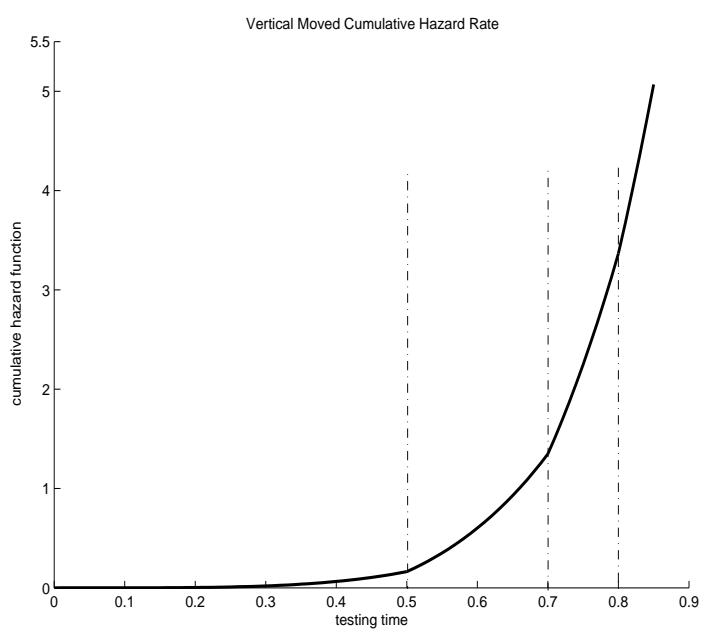

(b) Cumulative Hazard Function of SSALT

Fig. 1 Proportional Hazard Model

stress level $x_{i}$ through a $\log$ linear function, $\log \left(\theta_{i}\right)=\beta_{0}+\beta_{1} x_{i}, i=1, \ldots, k$, where the $\beta_{0}, \beta_{1}$ are unknown regression parameters. It is easily seen that the failure rate function of $\mathrm{KH}$ model is given by

$$
h(w)= \begin{cases}\delta \theta_{1} w^{\delta-1} & 0 \leq w<\tau_{1} \\ \delta \theta_{2} w^{\delta-1} & \tau_{1} \leq w<\tau_{2} \\ \cdots & \tau_{k-1} \leq w<\infty \\ \delta \theta_{k} w^{\delta-1} & \end{cases}
$$

This is a Weibull failure rate function at each stress with different scale parameters $\theta_{i}$ 's and a common shape parameter $\delta$. The ratio of two failure rates under two different stress levels is a constant over time since $h_{i}(w) / h_{j}(w)=\theta_{i} / \theta_{j}=e^{\beta_{1}\left(x_{i}-x_{j}\right)}$ with $h_{i}(w)=\delta \theta_{i} w^{\delta-1}, i=1, \ldots, k$. Hence, it has the desirable proportional hazard property as outlined in Lawless (2003), and we call it the Weibull proportional hazard model. Essentially, KH model relates the effect of physical stress to the hazard rate of the product. The cumulative failure rate of the Weibull $\mathrm{PH}$ model with $H_{i}(w)=\theta_{i} w^{\delta}, w>0$ is

$$
H(w)=\left\{\begin{array}{lr}
H_{1}(w) & 0 \leq w<\tau_{1} \\
H_{2}(w)-\left(H_{2}\left(\tau_{1}\right)-H_{1}\left(\tau_{1}\right)\right) & \tau_{1} \leq w<\tau_{2} \\
\cdots & \\
H_{k}(w)-\left(H_{k}\left(\tau_{k-1}\right)-H_{k-1}\left(\tau_{k-1}\right)\right)-\cdots-\left(H_{2}\left(\tau_{1}\right)-H_{1}\left(\tau_{1}\right)\right) & \tau_{k-1} \leq w<\infty
\end{array}\right.
$$

The function $H_{i}(w)$ is the cumulative hazard function of Weibull distribution under a constant stress. Mathematically, one may take the segments of $H_{i}(w)$ 's at their respective time intervals and vertically shift them to form a continuous cumulative hazard function for the Weibull PH SSALT model. An illustration of such function is shown in Figure 1.

In contrast, the $\mathrm{CE}$ model forms a composite failure distribution function by assuming the product lifetime is shifted at the time of stress level change such that the survival functions of the change time are the same under two different stress levels. For Weibull CE model, under the same time frame, the cdf of failure time is given by

$$
F(w)=1- \begin{cases}\exp \left\{-\theta_{1} w^{\delta}\right\} & 0 \leq w<\tau_{1} \\ \exp \left\{-\theta_{2}\left(w-\tau_{1}+s_{1}\right)^{\delta}\right\} & \tau_{1} \leq w<\tau_{2} \\ \cdots & \\ \exp \left\{-\theta_{k}\left(w-\tau_{k-1}+s_{k-1}\right)^{\delta}\right\} & \tau_{k-1} \leq w<\infty\end{cases}
$$




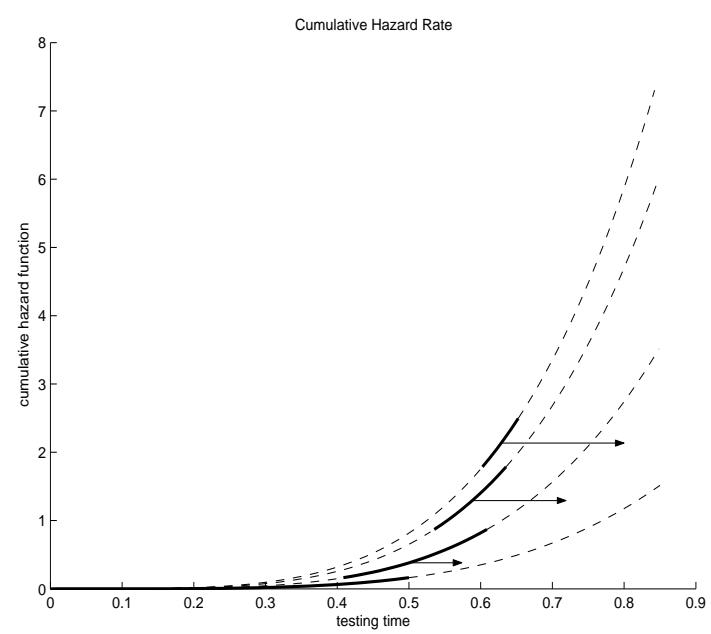

(a) Cumulative Hazard Functions of Constant-Stress ALTs

Fig. 2 Cumulative Exposure Model

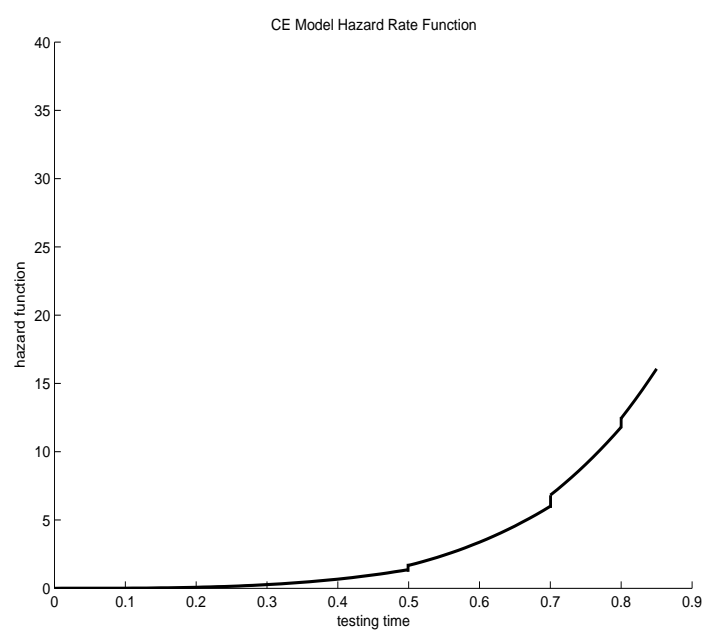

(a) CE Hazard Rate Function

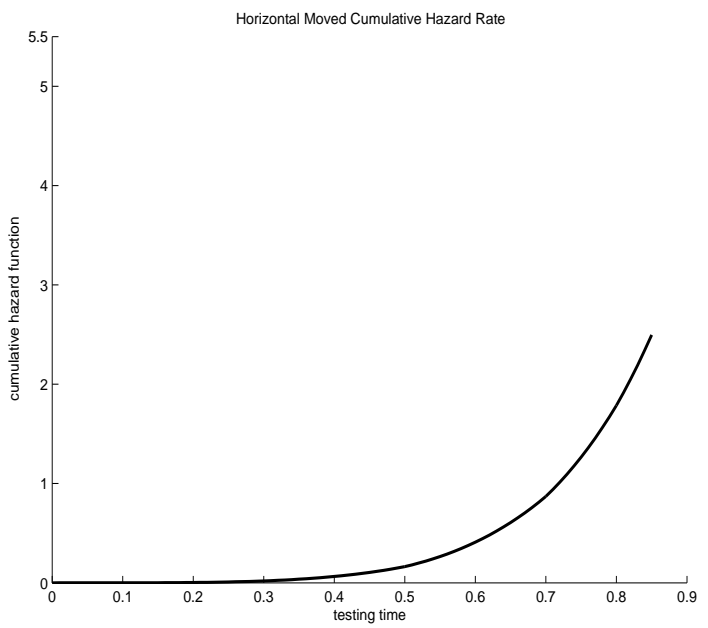

(b) Cumulative Hazard Function of SSALT

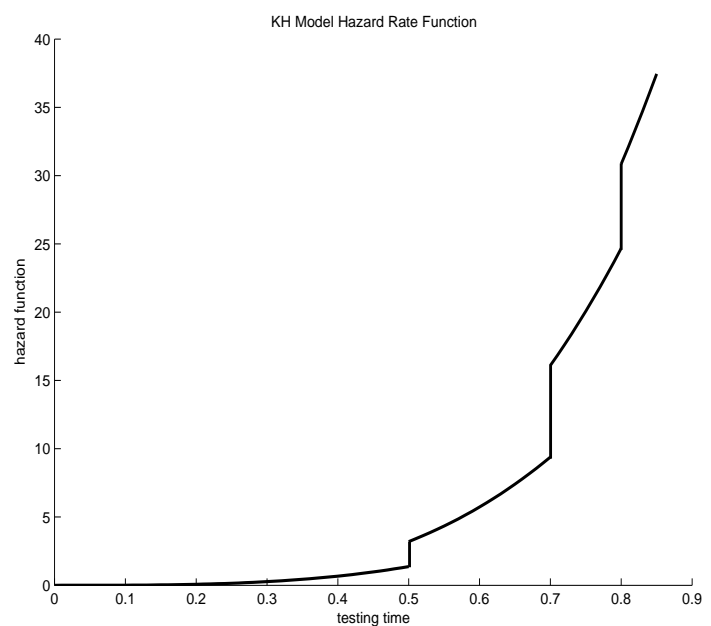

(b) PH Hazard Rate Function

Fig. 3 Hazard Rate Functions

where $s_{i}$ is an equivalent starting time under the $i$ th stress level satisfying $\theta_{i}\left(\tau_{i}-\tau_{i-1}+s_{i-1}\right)^{\delta}=\theta_{i+1} s_{i}^{\delta}, i=$ $1,2, \ldots, k-1$ with $s_{0}=0$ and $\tau_{0}=0$. The corresponding cumulative failure rate function is

$$
H(w)= \begin{cases}H_{1}(w) & 0 \leq w<\tau_{1} \\ H_{2}\left(w-\tau_{1}+s_{1}\right) & \tau_{1} \leq w<\tau_{2} \\ \ldots & \\ H_{k}\left(w-\tau_{k-1}+s_{k-1}\right) & \tau_{k-1} \leq w<\infty\end{cases}
$$

with $H_{i}(w)=\theta_{i} w^{\delta}, w>0$. The failure rate function at the $i$ th stress level becomes $h_{i}(w)=\delta \theta_{i}\left(w-\tau_{i-1}+\right.$ $\left.s_{i-1}\right)^{\delta-1}, \tau_{i-1} \leq w<\tau_{i}$. Graphically, for the Weibull CE model, the composite cumulative failure rate is made by horizontally shifting the individual cumulative hazard function segment as shown in Figure 
2; while in the Weibull PH model, the shift is made vertically as shown in Figure 1 . Their corresponding failure rate functions under the same time frame are displayed in Figure 3. When $\delta=1$, the failure time under each stress level is exponentially distributed and the curves of $H_{i}(w)$ become straight lines, so that the composite cumulative failure rates of the two models coincide with each other. Empirically, $\mathrm{PH}$ models correlate the effect of physical stress to the failure rate of the product while CE models to the failure time. As shown in Khamis and Higgins (1998), the Weibull PH model appears to be as flexible as the Weibull CE model in the fitting step-stress test data and has major computational advantages due to its mathematical simplicity. Moreover, the attractive mathematical properties will make Bayesian inference much convenient for the Weibull PH model than the Weibull CE model (presented in Dorp and Mazzuchi (2005)). Khamis and Higgins (1998) presented a simple Bayesian analysis in the case of known shape parameter $\delta$. Here we provide a "fully" Bayesian inference assuming all three parameters $\beta_{0}, \beta_{1}, \delta$ unknown in the following section.

\section{Bayesian Analysis}

\subsection{Likelihood Function}

Suppose that the observational data $\mathbf{D}=\left\{\left(w_{i j}, m_{i}\right), i=1, \ldots, k, j=1, \ldots, n_{i}\right\}$ with $w_{i j}$ being the $j^{\text {th }}$ failure time, $m_{i}$ the number of right censored units, and $n_{i}$ the number of failed units at the $i^{\text {th }}$ stress level. Let $n=\sum_{i=1}^{k} n_{i}$ and $m=\sum_{i=1}^{k} m_{i}$, so there are totally $n+m$ units initially placed on the test. The test starts with the first stress level $x_{1}$ and run until time $\tau_{1}$ when the stress level is changed to $x_{2}$, and so on. The test is completed on the stress level $x_{k}$ at the termination time $\tau_{k}$. With the Weibull PH model, the probability density function (pdf) is given by

$$
f(w)=\left\{\begin{array}{lc}
\delta \theta_{1} w^{\delta-1} \exp \left\{-\theta_{1} w^{\delta}\right\} & 0 \leq w<\tau_{1} \\
\delta \theta_{2} w^{\delta-1} \exp \left\{-\theta_{2}\left(w^{\delta}-\tau_{1}^{\delta}\right)-\theta_{1} \tau_{1}^{\delta}\right\} & \tau_{1} \leq w<\tau_{2} \\
\ldots & \\
\delta \theta_{k} w^{\delta-1} \exp \left\{-\theta_{k}\left(w^{\delta}-\tau_{k-1}^{\delta}\right)-\ldots-\theta_{2}\left(\tau_{2}^{\delta}-\tau_{1}^{\delta}\right)-\theta_{1} \tau_{1}^{\delta}\right\} & \tau_{k-1} \leq w<\tau_{k}
\end{array}\right.
$$

leading to the likelihood function $L\left(\theta_{1}, \ldots, \theta_{k}, \delta \mid \mathbf{D}\right)=\prod_{i=1}^{k}\left\{\prod_{j=1}^{n_{i}} f\left(w_{i j}\right)\left(S_{i}\left(\tau_{i}\right)\right)^{m_{i}}\right\}$ where the $i^{\text {th }}$ survival function $S_{i}\left(\tau_{i}\right)=\exp \left\{-\theta_{i}\left(\tau_{i}^{\delta}-\tau_{i-1}^{\delta}\right)-\ldots-\theta_{2}\left(\tau_{2}^{\delta}-\tau_{1}^{\delta}\right)-\theta_{1} \tau_{1}^{\delta}\right\}$. By introducing the log-linear function of $\theta_{i}, \log \left(\theta_{i}\right)=\beta_{0}+\beta_{1} x_{i}$, it becomes

$$
\begin{aligned}
L\left(\beta_{0}, \beta_{1}, \delta \mid \mathbf{D}\right)= & \delta^{n} \exp \left\{n \beta_{0}+\left(\sum_{i=1}^{k} n_{i} x_{i}\right) \beta_{1}-\sum_{i=1}^{k} e^{\beta_{0}+\beta_{1} x_{i}} u_{i}(\delta)\right\} \\
& \times \prod_{i=1}^{k} \prod_{j=1}^{n_{i}} w_{i j}^{\delta-1}
\end{aligned}
$$

where $u_{i}(\delta)=\sum_{j=1}^{n_{i}}\left(w_{i j}^{\delta}-\tau_{i-1}^{\delta}\right)+\left(n+m-\sum_{l=1}^{i} n_{l}-\sum_{l=1}^{i-1} m_{l}\right)\left(\tau_{i}^{\delta}-\tau_{i-1}^{\delta}\right), i=1,2, \ldots, k-1$ with $\tau_{0}=0$, and $u_{k}(\delta)=\sum_{j=1}^{n_{k}}\left(w_{k j}^{\delta}-\tau_{k-1}^{\delta}\right)+m_{k}\left(\tau_{k}^{\delta}-\tau_{k-1}^{\delta}\right)$. This likelihood function is applicable to type-I censored (Bai et al., 1989), type-II censored (Xiong, 1998) and right censored testings. If we treat $w_{i j}^{\delta}$ and $\tau_{i}^{\delta}$ as the transformed failure time and censoring time, respectively, then $u_{i}(\delta)$ is the total transformed testing time of all test units experienced at the $i^{\text {th }}$ stress level. In fact, if $\delta$ is known and let $v=w^{\delta}$, then $v$ will be exponentially distributed at each stress level with failure rate of $\theta_{i}$ and the likelihood function is exactly the same as that of an exponential SSALT. 


\subsection{Bayesian Inference Procedure}

Now we present a Bayesian inference for the Weibull PH Model. We adopt independent priors for the parameters $\beta_{0}, \beta_{1}$ and $\delta$. The joint posterior density function will be proportional to the product of the likelihood function and the priors

$$
f\left(\beta_{0}, \beta_{1}, \delta \mid \mathbf{D}\right) \propto L\left(\beta_{0}, \beta_{1}, \delta \mid \mathbf{D}\right) f\left(\beta_{0}\right) f\left(\beta_{1}\right) f(\delta)
$$

From the functional form of the posterior distribution, we notice that the conjugate prior of $\beta_{0}$ is a $\log$-Gamma $\left(\kappa_{0}, \gamma_{0}\right)$ or generalized extreme value distribution with the hyper-parameters shape $\kappa_{0}$, and scale $\gamma_{0}$, i.e. $f\left(\beta_{0}\right) \propto \exp \left\{\kappa_{0} \beta_{0}-\gamma_{0} e^{\beta_{0}}\right\}$. It results in a $\log \operatorname{Gamma}\left(\kappa_{0}^{*}, \gamma_{0}^{*}\right)$ for the conditional posterior of $\beta_{0}$

$$
f\left(\beta_{0} \mid \beta_{1}, \delta, \mathbf{D}\right) \propto L\left(\beta_{0} \mid \beta_{1}, \delta, \mathbf{D}\right) f\left(\beta_{0}\right) \propto \exp \left\{\kappa_{0}^{*} \beta_{0}-\gamma_{0}^{*} e^{\beta_{0}}\right\}
$$

with the updated shape $\kappa_{0}^{*}=\kappa_{0}+n$ and scale $\gamma_{0}^{*}=\gamma_{0}+\sum_{i=1}^{k} e^{\beta_{1} x_{i}} u_{i}(\delta)$. There are no conjugate priors existed for the other two parameters $\beta_{1}$ and $\delta$. However, we notice that the conditional likelihood of $\beta_{1}$ is

$$
L\left(\beta_{1} \mid \beta_{0}, \delta, \mathbf{D}\right) \propto \exp \left\{\left(\sum_{i=1}^{k} n_{i} x_{i}\right) \beta_{1}-e^{\beta_{0}} \sum_{i=1}^{k} u_{i}(\delta) e^{x_{i} \beta_{1}}\right\}
$$

Hence we may consider a prior of $\beta_{1}$ having a "similar" form as its conditional likelihood function, and choose $\beta_{1}=\frac{\log (Z)}{\eta_{1}}$ where $Z \sim \operatorname{Gamma}\left(\frac{\kappa_{1}}{\eta_{1}}, \gamma_{1}\right)$ with the hyper-parameters $\kappa_{1}, \eta_{1}, \gamma_{1}$. So the prior density function $f\left(\beta_{1}\right) \propto \exp \left\{\kappa_{1} \beta_{1}-\gamma_{1} e^{\eta_{1} \beta_{1}}\right\}$ and the full conditional posterior becomes

$$
\begin{aligned}
f\left(\beta_{1} \mid \beta_{0}, \delta, \mathbf{D}\right) & \propto L\left(\beta_{1} \mid \beta_{0}, \delta, \mathbf{D}\right) f\left(\beta_{1}\right) \\
& \propto \exp \left\{\left(\kappa_{1}+\sum_{i=1}^{k} n_{i} x_{i}\right) \beta_{1}-\left(\gamma_{1} e^{\eta_{1} \beta_{1}}+e^{\beta_{0}} \sum_{i=1}^{k} u_{i}(\delta) e^{x_{i} \beta_{1}}\right)\right\}
\end{aligned}
$$

Similarly, with considering $\delta>0$, we choose $\delta=\frac{\log (Z)}{\eta_{2}}$ where $Z \sim \operatorname{truncated}$ Gamma $\left(\frac{\kappa_{2}}{\eta_{2}}, \gamma_{2}\right)$ with $Z>1$ and the hyper-parameters $\kappa_{2}, \eta_{2}, \gamma_{2}$, so that the prior $f(\delta) \propto \exp \left\{\kappa_{2} \delta-\gamma_{2} e^{\eta_{2} \delta}\right\}$ with truncation $\delta>0$. Thus the conditional posterior is

$$
\begin{aligned}
f\left(\delta \mid \beta_{0}, \beta_{1}, \mathbf{D}\right) & \propto L\left(\delta \mid \beta_{0}, \beta_{1}, \mathbf{D}\right) f(\delta) \\
& \propto \delta^{n} \exp \left\{\left(\kappa_{2}+\sum_{i=1}^{k} \sum_{j=1}^{n_{i}} \log \left(w_{i j}\right)\right) \delta-\left(\gamma_{2} e^{\eta_{2} \delta}+\sum_{i=1}^{k} e^{\beta_{0}+\beta_{1} x_{i}} u_{i}(\delta)\right)\right\}
\end{aligned}
$$

In practice, the vague or noninformative log-Gamma priors can be chosen with smaller hyperparameter values for the shape $\left(\frac{\kappa_{i}}{\eta_{i}}\right)$ and scale $\left(\gamma_{i}\right)$ so that it will have little influence on the posterior distributions. The posterior inference can be made through the joint posterior samples $\left(\beta_{0}, \beta_{1}, \delta\right)$, which will be drawn through a Markov chain Monte Carlo (MCMC) technique (Gilks et al., 1996). In particular, here we employ a Gibbs sampling algorithm through their full conditional distributions in (9), (11) and (12). Although the last two conditional posteriors are not "standard" distributions, they are log-concave functions. The implementation of efficient Adaptive Rejection Sampling (ARS) technique (Gilks and Wild, 1992) can be utilized within Gibbs sampling procedure. The technique is a type of rejection sampling using a sequence of envelope and squeezing functions which converge to the target density function as sampling proceeds. It is also adaptive since the envelope and squeezing functions are constructed at previously sampled points. See the algorithm details in Gilks and Wild (1992). 
Table 1 Simulated Data: Prespecified Values

\begin{tabular}{|c|l|l|c|}
\hline $\begin{array}{c}\text { Stress Number } \\
k\end{array}$ & $\begin{array}{c}\text { Failures at Each Stress } \\
n_{i}\end{array}$ & $\begin{array}{c}\text { Stress Changing Times } \\
\tau_{i}\end{array}$ & $\begin{array}{c}\text { Terminated Time } \\
\tau_{k}\end{array}$ \\
\hline 3 & $6,3,9$ & $0.5,2.0$ & 3.5 \\
4 & $9,14,5,12$ & $0.5,2.0,3.5$ & 5.0 \\
5 & $15,6,3,1,10$ & $0.5,2.0,3.5,5.0$ & 8.0 \\
6 & $12,6,9,2,1,8$ & $0.5,2.0,3.5,5.0,8.0$ & 10.0 \\
\hline
\end{tabular}

Table 2 Simulated Data: Parameters Estimation with True Values $\beta_{0}=-2.85, \beta_{1}=-1, \delta=2.97$

\begin{tabular}{|c|l|l|}
\hline Stress Number $k$ & Posterior Estimates & 95\% Credible Intervals \\
\hline 3 & $\hat{\beta}_{0}=-3.10$ & $(-3.55,-2.60)$ \\
& $\hat{\beta}_{1}=-0.94$ & $(-1.14,-0.85)$ \\
& $\hat{\delta}=2.93$ & $(2.84,3.08)$ \\
\hline 4 & $\hat{\beta}_{0}=-3.06$ & $(-3.49,-2.66)$ \\
& $\hat{\beta}_{1}=-0.93$ & $(-1.10,-0.89)$ \\
& $\hat{\delta}^{2.99}$ & $(2.88,3.03)$ \\
\hline 5 & $\hat{\beta}_{0}=-3.02$ & $(-3.38,-2.71)$ \\
& $\hat{\beta}_{1}=-0.97$ & $(-1.04,-0.93)$ \\
& $\hat{\delta}=2.96$ & $(2.95,2.98)$ \\
\hline \multirow{2}{*}{6} & $\hat{\beta}_{0}=-2.95$ & $(-3.30,-2.74)$ \\
& $\hat{\beta}_{1}=-0.98$ & $(-1.02,-0.92)$ \\
& $\hat{\delta}=2.98$ & $(2.96,2.98)$ \\
\hline
\end{tabular}

\section{Applications}

\subsection{Simulation Study}

We first investigate the performance of the proposed method using simulated datasets from the Weibull PH model with the parameter values $\beta_{0}=-2.85, \beta_{1}=-1$ and $\delta=2.97$. The simulated data contain $k=3,4,5,6$ stresses, in which the number of failures $n_{i}$ at each stress generated from the discrete uniform $(1,15)$, the time points of stresses changed and the pre-specified termination time listed in Table 1. The failure times $w_{i j}$ at $i^{\text {th }}$ stress level were generated from the Weibull distribution with the log-scale function $\log \left(\theta_{i}\right)=\beta_{0}+\beta_{1} x_{i}, i=1,2, \ldots, k$, where the stress levels $x_{i}$ are generated from $\mathcal{N}(2,1)$. For the Bayesian analysis, we adopt somehow vague but proper prior distributions with hyperparameter values chosen as $\kappa_{0}=\kappa_{1}=\kappa_{2}=0.001, \gamma_{0}=\gamma_{1}=\gamma_{2}=0.001, \eta_{0}=\eta_{1}=\eta_{2}=1$. For each simulated data, we run five MCMC chains with fairly different initial values and each with 500 burn in iterations followed by 1,000 sample iterations. The scale reduction factor estimate $\sqrt{\widehat{R}}=\sqrt{\frac{\operatorname{Var}(\psi)}{W}}$ is used to monitor convergence of MCMC simulations (Gelman et al., 1996), where $\psi$ is the estimand of interest, $\operatorname{Var}(\psi)=\frac{n-1}{n} W+\frac{1}{n} B$ with the iteration number $n$ for each chain, the between- and within-sequence variances $B$ and $W$. The scale factors for the sequences of $\beta_{0}, \beta_{1}$ and $\delta$ are within 1.00-1.02 for all five MCMC chains, indicating their convergence. To provide relatively independent samples for improvement of prediction accuracy, we calculated the posterior estimates by the means of every $5^{\text {th }}$ sampled values after discarding the first 500 iterations for all five MCMC chains. The posterior results, summarized in Table 2, clearly show that the estimation precision increases as the number of stresses $k$ increases, and that $95 \%$ credible intervals (Eberly and Casella, 2003) are relative narrow, especially for the estimation of $\beta_{1}$ and $\delta$. 
Table 3 Test Data on Cable Insulation

\begin{tabular}{|c|c|l|}
\hline Kilovolts & Final Step & \multicolumn{1}{|c|}{ Failure or Suspension $(+)$ Time $(\min )$} \\
\hline 26.0 & 1 & $363.9+, 898.4+$ \\
28.5 & 2 & 1160.0 \\
31.0 & 3 & $1962.9,2460.9,2460.9+, 2700.4$ \\
33.4 & 4 & 2923.9 \\
36.0 & 5 & $102,113,113,1096.9,1097.9,4142.1$ \\
38.5 & 6 & $345,345+, 370,1249,1250.8,1333,1333+$ \\
\hline
\end{tabular}

Table 4 Estimation Results for the Cable Data

\begin{tabular}{|c|ll|l|}
\hline Parameter & \multicolumn{2}{|c|}{ MLE (95\% Confidence Intervals) } & \multicolumn{1}{c|}{$\begin{array}{c}\text { Bayes PH Model } \\
\text { (95\% Credible Intervals) }\end{array}$} \\
\hline$\delta$ & $0.76(0.18,1.33)$, & PH Model & $0.72(0.15,1.31)$ \\
$\beta_{0}$ & $-111.37(-195.93,-26.51)$, & $-113.56(-196.25,-28.43)$ & $-120.73(-173.51,-42.12)$ \\
$\beta_{1}$ & $15.07(3.59,26.52)$, & $14.98(3.65,26.07)$ & $12.55(5.80,22.69)$ \\
\hline
\end{tabular}

\subsection{Real Data Analysis}

We apply the proposed Bayesian method to two real SSALT datasets. The first data is from a stepstress test of cable insulation described in Nelson (1980). The test was run to estimate life of cable at a design stress of 400 volts/mil. Table 3 shows the pattern of specified stresses (Kilovolts), final step and total time on tested specimens. We adopt diffused priors with hyperparameter values $\kappa_{0}=\kappa_{1}=$ $\kappa_{2}=0.01, \gamma_{0}=\gamma_{1}=\gamma_{2}=0.01, \eta_{0}=\eta_{1}=\eta_{2}=1$, and run five MCMC chains wth different starting values. In this case 20,000 iterations were sufficient for convergence since the Gelman-Rubin statistic $\sqrt{\widehat{R}}$ for all three parameters are close to 1 . The parameter values are estimated as the means of every $5^{\text {th }}$ sampled values after discarding first 5,000 iterations. These posterior estimates are close to the maximum likelihood estimates (MLE) obtained from the Weibull PH model, as summarized in Table 4. For the purpose of comparison, in this table, we also report the MLE and large-sample based $95 \%$ confidence intervals (Meek and Escobar, 1995) for each parameter for both Weibull CE model (Nelson, 1980) and PH model as well as the $95 \%$ credible intervals by the Bayesian approach (Eberly and Casella, 2003). Both frequentist and Bayesian approaches provide similar parameter estimation values, but the credible intervals obtained from the Bayesian approach are a little narrower than the confidence intervals from the frequentist approach.

The second SSALT data is from degradation experiments of light emitting diodes (LED) described in Zhao and Elsayed (2005). It is known that high temperature and high humidity can significantly shorten the lifetime of LED. The experiment was conducted at four temperature levels under a constant humidity level. The failure data were used for predicting the products lifetime under the normal use temperature, $50^{\circ} \mathrm{C}$. The test was terminated at the time of 720 hours and some test units are removed (right censored) at the times of changing temperature due to various reasons. The experimental condition and lifetime data are summarized in Table 5.

According to the Arrhenius model of reliability testing with temperature, the natural stress variable is the reciprocal of the temperature in degree Kelvin, i.e. $x_{i}=1 / T_{i}$. For this analysis, we set $x_{i}=323 / T_{i}$ to normalize the stress by making $x=1$ at $T=323\left(50^{\circ} \mathrm{C}\right)$. As previously, the vague priors with hyperparameter values $\kappa_{0}=\kappa_{1}=\kappa_{2}=0.01, \gamma_{0}=\gamma_{1}=\gamma_{2}=0.01, \eta_{0}=\eta_{1}=\eta_{2}=1$ are chosen, and five MCMC chains were run with different starting values. The parameter values are estimated as the means of every $5^{\text {th }}$ sampled values after discarding first 5,000 iterations. Table 6 listed MLE and interval estimates for each parameter as well as the posterior estimates and credible intervals by the Bayesian PH model. It is observed that with noninformative priors, the Bayesian approach provides the comparable estimation 
Table 5 LED Testing Conditions and Lifetime Data

\begin{tabular}{|c|c|l|}
\hline $\begin{array}{c}\text { Temperature } \\
\text { (in degree Kelvin) }\end{array}$ & $\begin{array}{c}\text { Testing Period } \\
\text { (in hours) }\end{array}$ & \multicolumn{1}{|c|}{ Failure or Suspension (+) Time } \\
\hline 363 & $(0,300)$ & $300+$ \\
413 & $(300,500)$ & $347,397,432,491,500+, 500+$ \\
433 & $(500,600)$ & $512,567,574,588,597,600+, 600+$ \\
448 & $(600,720)$ & $603,605,615,633,634,637,644,653,675,684,699$, \\
& & $706,718,720,720+, 720+, 720+, 720+$ \\
\hline
\end{tabular}

Table 6 Estimation Results for the LED Data

\begin{tabular}{|c|c|c|}
\hline Parameter & $\begin{array}{c}\text { MLE (95\% Confidence Intervals) } \\
\text { CE Model } \quad \text { PH Model }\end{array}$ & $\begin{array}{c}\text { Bayes PH Model } \\
\text { (95\% Credible Intervals) }\end{array}$ \\
\hline $\bar{\delta}$ & $6.81(4.03,9.59)$, & $4.91(4.52,5.61)$ \\
\hline$\beta_{0}$ & $-20.73(-34.74,-6.72),-22.18(-36.13,-8.23)$ & $-23.29(-26.17,-18.76)$ \\
\hline$\beta_{1}$ & $-15.06(-34.36,4.24),-16.34(-35.25,2.57)$ & $-11.70(-17.80,-7.86)$ \\
\hline
\end{tabular}

results as frequentist approach with a little narrower interval estimation. In fact, one can see that the $95 \%$ confidence interval of $\beta_{1}$ is large enough to include zero which indicates that the effect of the stress cannot be identified at this significant level. This difficulty does not appear in Bayesian inference, even with the vague prior distributions. Therefore, in this case, the reliability prediction can be improved by the Bayesian approach. We run another chain with relatively informative priors by choosing the hyper-parameters $\kappa_{0}=$ $\kappa_{1}=\kappa_{2}=0.1, \gamma_{0}=\gamma_{1}=\gamma_{2}=0.1, \eta_{0}=\eta_{1}=\eta_{2}=1$, the estimates become $\hat{\delta}=4.83, \hat{\beta}_{0}=-23.61, \hat{\beta}_{1}=$ -12.08 , and $95 \%$ credible intervals are $(4.57,5.50),(-25.18,-20.06),(-18.27,-8.85)$, respectively. Not surprisingly, it leads to narrower intervals for the parameter estimations.

\section{Conclusions}

In this article we presented a Bayesian approach for Weibull PH model for SSALT data analysis and demonstrated the key difference between this model and the traditional Weibull CE model. The Weibull PH model directly links the effect of physical stress to the product failure rate, and avoids the model complexity caused by time transformation in a cumulative fashion. The formulation of Weibull PH model makes posterior inference mathematically simpler than Weibull CE model without sacrificing flexibility for fitting data. The prior distributions we adopted result in a conjugate prior for the intercept parameter in the log-linear life-stress function, and log-concavity of conditional posteriors for other parameters, making the Bayesian analysis very convenient and efficient. Oftentimes reliability testing generates limited number of failure time data, which causes extremely flat likelihood function and large uncertainty in parameter estimation. Bayesian inference can overcome this difficulty, especially when some prior information on the acceleration model can be acquired from existing studies or from engineering experience. The examples showed the convenience and efficiency of MCMC algorithm in the posterior inference.

\section{Acknowledgement}

This research is partially supported by NSF/CMMI-0654417. Sha's work was also partially supported by Shanghai High Education 085 Project Fund. 


\section{References}

Bai DS, Kim MS and Lee SH (1989). Optimum simple step-stress accelerated life tests with censoring. IEEE Transactions on Reliability, 38(5), 528-532.

Cox D (1972). Regression models and life tables. Journal of the Royal Statistical Society, Series B, 34, $187-220$.

DeGroot MH and Goel PK (1979). Bayesian estimation and optimal designs in partially accelerated life testing. Naval Research Logistic Quarterly, 26, 223-235.

Doksum KA and Hoyland A (1991). Models for variable-stress accelerated life testing experiments based on Wiener process and inverse Gaussian distribution. Technometrics, 34, 74-82.

Eberly LE and Casella G (2003). Estimating Bayesian credible intervals. Journal of Statistical Planning and Inference, 112, 115-132.

Gelman A, Carlin JB, Stern HS and Rubin DB (1996). Bayesian Data Analysis, London: Chapman \& Hall.

Gilks WR and Wild P (1992). Adaptive rejection sampling for Gibbs sampling. Applied Statistics, 41(2), $337-348$.

Gilks WR, Richardson S and Spiegelhalter DJ (eds.). (1996). Markov chain Monte Carlo in Practice, London: Chapman \& Hall.

Khamis IH and Higgins JJ (1998). A new model for step-stress testing. IEEE Transactions on Reliability, $47(2), 131-134$.

Lawless JF (2003). Statistical Models and Methods for Lifetime Data, 2nd edition, New Jersey: John Wiley \& Sons.

Lee J and Pan R (2008). Bayesian inference model for step-stress accelerated life testing with type-II censoring. 2008 Proc. Ann. Reliability \& Maintainability Symp., IEEE. Las Vegas, NV.

Meek WQ and Escobar LA (1995). Teaching about approximate confidence regions based on maximum likelihood estimation. The American Statistician, 49(1), 48-53.

Nelson WB (1980). Accelerated life testing step-stress models and data analysis. IEEE Transactions on Reliability, 29(3), 103-108.

Van Dorp JR and Mazzuchi TA (2004). A general Bayes exponential inference model for accelerated life testing. Journal of Statistical Planning and Inference, 119, 54-74.

Van Dorp JR and Mazzuchi TA (2005). A general Bayes Weibull model for accelerated life testing. Reliability Engineering and System Safety, 90, 140-147.

Wang R and Fei H (2004). Conditions for the coincidence of the TFR, TRV and CE models. Statistical Papers, 45(3), 393-412.

Xiong C (1998). Inference on a simple step-stress model with type-II censored exponential data. IEEE Transactions on Reliability, 47(2), 142-146.

Zhao W and Elsayed EA (2005). A general accelerated life model for step- stress testing. IIE Transactions, 37(11), 1059-1069. 